\title{
Investigation of Surgery Characteristics in Patients with Spondylolisthesis, Rasht, Iran from 2006 to 2011
}

\author{
Shahrokh Yousefzadeh-Chabok ${ }^{1}$, Ehsan Kazemnejad-Leili ${ }^{2}$, Seyed-Morteza Khatami ${ }^{3}$, Leila \\ Kouchakinejad-Eramsadati ${ }^{4}$, Babak Alijani ${ }^{5}$ * \\ ${ }^{1}$ MD, Professor of Neurosurgery, Guilan Road Trauma Research Center, Poursina Hospital, Guilan University of Medical Sciences, Rasht, \\ Guilan, Iran \\ ${ }^{2} \mathrm{PhD}$, Associate Professor of Biostatistics, Guilan Road Trauma Research Center, Guilan University of Medical Sciences, Rasht, Guilan, Iran \\ ${ }^{3}$ MD, Guilan Road Trauma Research Center, Guilan University of Medical Sciences, Rasht, Guilan, Iran \\ ${ }^{4}$ BSc in Nursing, MA in Human Resource Management, Guilan Road Trauma Research Center, Guilan University of Medical Sciences, \\ Rasht, Guilan, Iran \\ ${ }^{5}$ MD, Assistant Professor of Neurosurgery, Guilan Road Trauma Research Center, Guilan University of Medical Sciences, Rasht, Guilan, \\ Iran \\ * Corresponding Author: Poursina Hospital, Guilan University of Medical Sciences, Rasht, Guilan, Iran. Tel: +981333338373, Fax: \\ +981333338373. E-mail:1kouchaki@gmail.com
}

Article Type: Research Article

Received: November 5, 2015, Last revised: July 5, 2015, Accepted: September 22, 2015

\section{Abstract}

Background \& Aim: Spondylolisthesis is a visible deformity in lumbosacral region with vertebral slip and fracture or other deformities of pars interarticularis. This study aims at investigating the characteristics of spondylolisthesis surgery in operated patients.

Methods \& Materials/Patients: This is a retrospective study which included all the patients who had undergone spondylolisthesis surgery by one same surgeon from 2006 to 2011. Demographic characteristics such as age, gender, and surgery information including type of spondylolisthesis, incision site, laminectomy site, foraminotomy site, fixation site of vertebra and site of vertebral disc evacuation were collected. The data were analyzed using SPSS (Version16).

Results: 52 of 63 enrolled patients were women $(82.5 \%)$ and 11 men $(17.5 \%)$ with mean age of 49.6 years. Based on frequency, spondylolisthesis types were 60 degenerative $(95.2 \%), 2$ post-surgical $(3.2 \%)$ and 1 post-traumatic $(1.6 \%)$, respectively. 58 patients had spondylolisthesis only in one vertebra and 5 patients in two vertebrae. The total number of slipped vertebrae was 68, in which slip was seen in L3, L4 or L5 vertebrae. In 42 patients, laminectomy was performed only in one vertebra, in 19 patients in two vertebrae, in 1 patient in three vertebrae and in 1 patient in five vertebrae. The total number of vertebrae with foraminotomy was 106. The highest frequency of foraminotomy was seen in L5, L4, and $\mathrm{S} 1$ vertebrae, respectively. Except in one case, the rest 62 patients underwent fixation surgery in two or three vertebrae.

Conclusion: Based on the results, the frequency of spondylolisthesis was approximately five times in women compared to men. The most common type was degenerative spondylolisthesis. Type of surgery was fixation with pedicle screw and fusion.

Keywords: Surgery; Characteristics; Spondylolisthesis

Please cite this paper as: Yousefzadeh-Chabok Sh, Alijani B, Kazemnejad-Leili E, Khatami S.M, Kouchakinejad-Eramsadati L. Investigation of Surgery Characteristics in Patients with Spondylolisthesis, Rasht, Iran from 2006 to 2011.Iran. J. Neurosurg. 2015;1(2):36-39.

\section{Introduction}

The term Spondylolisthesis stems from the Greek words Spondylos and olisthesis (1,2). It was first proposed by Taillard in 2008 as forward slipping of vertebra body along with pedicle, transverse process and upper articular process which is caused by fracture or elongation of pars interarticularis (3). It is generally a disorder characterized by a visible deformity in lumbosacralis, vertebral slip and fracture or other deformities of pars interarticularis (4). First case of spondylolisthesis was explained by a gynecologist called Herbinaux in 1772 in which the slip of L5 on sacrum had caused problems in a patient for childbirth delivery (5). Studies have reported the prevalence of spondylolisthesis $4 \%$ to $6 \%$ in the society (6). Types of spondylolisthesis according to Wiltse et.al. (2009) are as follows: 1) dysplastic, due to dysplasia of congenital articular processes, 2) isthmic, due to Pars articularis deficit 3) degenerative, resulting from degenerative changes in articular surfaces 4) traumatic, due to fracture of neural arch more than pars interarticularis 5) pathologic, due to weakness of the neural arch and bone disorders and 6) iatrogenic, caused by the removal of bone following bone decompression $(7,8)$. Spondylolisthesis may be 
Table 1. Frequency and Percentage of Slipped Vertebrae based on Sex

\begin{tabular}{|c|c|c|c|}
\hline Slipped Vertebra & $\begin{array}{c}\text { Total number } \\
\text { of Patients }\end{array}$ & Women (\%) & Men (\%) \\
\hline $\begin{array}{c}\text { L3 } \\
\text { spondylolisthesis }\end{array}$ & $5(7.9 \%)$ & $5(9.6 \%)$ & 0 \\
\hline $\begin{array}{c}\text { L4 } \\
\text { spondylolisthesis }\end{array}$ & $33(52.4 \%)$ & $29(55.8 \%)$ & $4(36.4 \%)$ \\
\hline $\begin{array}{c}\text { L5 } \\
\text { spondylolisthesis }\end{array}$ & $20(31.7 \%)$ & $14(26.9 \%)$ & $6(54.5 \%)$ \\
\hline $\begin{array}{c}\text { L3 \& L4 } \\
\text { spondylolisthesis }\end{array}$ & $2(3.2 \%)$ & $2(3.8 \%)$ & 0 \\
\hline $\begin{array}{c}\text { L4 \& L5 } \\
\text { spondylolisthesis }\end{array}$ & $3(4.8 \%)$ & $2(3.8 \%)$ & $1(9.1 \%)$ \\
\hline Total & $63(100 \%)$ & $52(100 \%)$ & $11(100 \%)$ \\
\hline
\end{tabular}

completely asymptomatic (9). Back pain is the most common clinical manifestation of spondylolisthesis (10). In fact, forward bend increases and backward bend reduces the pain. Many people, even with remarkable vertebral slip may remain healthy with no complication and pain complaints in their lifetime. Yet, others with a mild slip may suffer severe pain. Some people even with acute dislocation may be asymptomatic over time (1). Treatment of patients with spondylolisthesis can be conservative or surgical. Prior research report that indications of surgical treatment include uncontrollable pain, neurological deficits, and more than $50 \%$ spondylolisthesis in initial evaluation or about $25 \%$ to $50 \%$ progressive increase (11). Most patients respond well to conservative treatment (4). This study aimed at investigating the characteristics of spondylolisthesis surgery in operated patients in a teaching hospital in Iran.

\section{Methods and Materials/Patients}

This is a retrospective study which included all the patients who had undergone spondylolisthesis surgery from 2006 to 2011 by one same surgeon whose records were available in Medical Records Department of Poursina Hospital in Rasht, Guilan (North of Iran). The patients with no surgery records were excluded. Then a questionnaire was used to record demographic

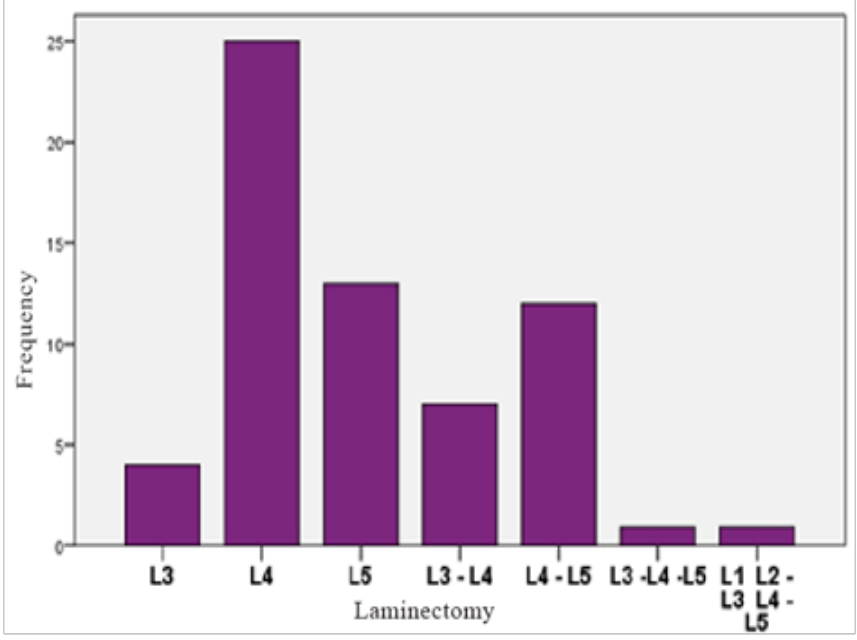

Figure 1. Frequency of Laminectomy in Patients with Spondylolisthesis Surgery
Table 2. Frequency of Foraminotomy Types

\begin{tabular}{|c|c|} 
Foraminotomy Types & Frequency (\%) \\
\hline L3 & $1(1.6)$ \\
\hline L4 & $3(4.8)$ \\
\hline L5 & $15(23.8)$ \\
\hline S1 & $4(6.3)$ \\
\hline L3-L4 & $1(1.6)$ \\
\hline L4-L5 & $22(34.9)$ \\
\hline L5-S1 & $14(22.2)$ \\
\hline L3-L4-L5 & $1(1.6)$ \\
\hline L4-L5-S1 & $1(1.6)$ \\
\hline L3-L4-L5-S1 & $1(1.6)$ \\
\hline & \\
\hline Total & $63(100)$ \\
\hline
\end{tabular}

characteristics such as age, sex and surgery information such as spondylolisthesis type, incision site, laminectomy site, foraminotomy site, site of vertebra fixation and site of disc evacuation. The data were analyzed using descriptive statistics using SPSS (Version 16).

\section{Results}

Of 63 patients enrolled in this study, 52 patients were women $(82.5 \%)$ and 11 men $(17.5 \%)$ (Mean age=49.6 years, $\mathrm{SD}=9.8$ ). The youngest and the oldest ones were 27 and 76 years old, respectively. Types of spondylolisthesis in these patients based on frequency were 49 degenerative $(77.7 \%), 11$ ischemic $(17.5 \%), 2$ post-surgical $(3.2 \%)$ and 1 traumatic (1.6\%). Based on the diagnosis in the surgery records, 58 patients $(92.06 \%)$ had spondylolisthesis only in one vertebra and 5 patients in two vertebrae (7.94\%). The total number of slipped vertebrae was 68. Slip was seen in L3, L4 and L5. The most common slipped vertebrae were L4 (55.9\%) and L5 (54.5\%) in women and men, respectively (Table1). 42 patients had laminectomy only in one vertebra, 19 patients in two vertebrae, 1 patient in three vertebrae and 1 patient in 5 vertebrae. The total number of vertebrae with laminectomy was 88. L4 (52.3\%) was the most frequent vertebra with laminectomy (Figure 1). In these

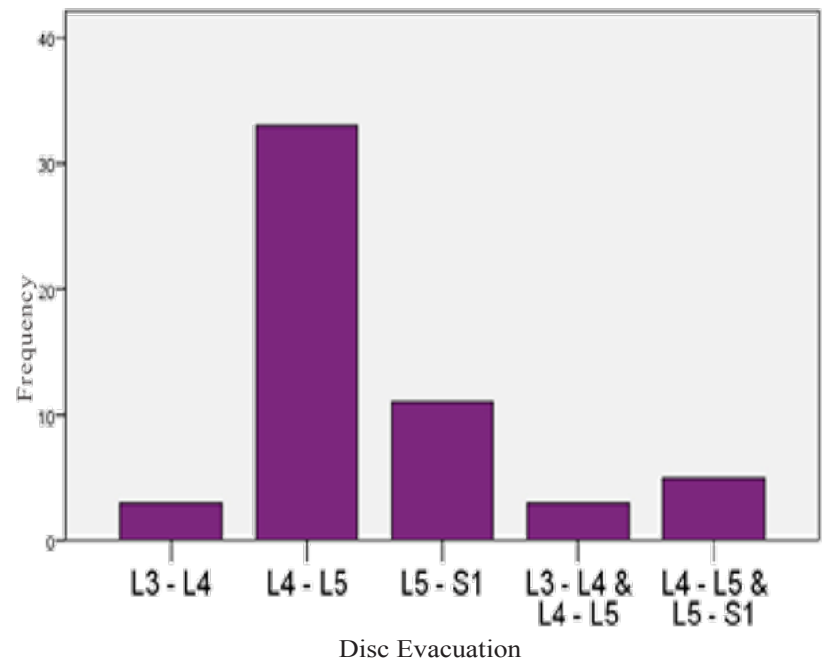

Figure 2. Frequency of Types of Disc Evacuation in Patients with Spondylolisthesis Surgery 
Table 3. Frequency of Fixation Types in Patients with Spondylolisthesis Surgery

\begin{tabular}{|c|c|}
\hline Fixation & $\mathbf{N}(\%)$ \\
\hline L3 - L4 & $4(6.5)$ \\
\hline L3 - L5 & $1(1.6)$ \\
\hline L4 - L5 & $17(27.4)$ \\
\hline L5 - S1 & $13(21.0)$ \\
\hline L1 - L2 - L3 & $1(1.6)$ \\
\hline L2 - L3 - L4 & $2(3.2)$ \\
\hline L3 - L4 - L5 & $14(22.6)$ \\
\hline L3 - L4 - S1 & $1(1.6)$ \\
\hline L2 - L4 - L5 & $1(1.6)$ \\
\hline L4 - L5 - S1 & $8(12.9)$ \\
\hline Total & $62(100)$ \\
\hline
\end{tabular}

63 patients, foraminotomy was done only on one vertebra in 23 patients, on two vertebrae in 37 patients and on three vertebrae in 3 patients. Total number of vertebrae foraminotomy in this group equaled to 106 vertebrae among which the highest frequency of this figure was observed in 54 cases of L5 (50.5\%), 29 cases of L4 (27.1\%), 20 cases of S1 (18.7\%), and 4 cases of L3 (3.7\%), respectively (Table 2 ). Except one case, other patients underwent fixation in one or two vertebrae. 35 patients had fixation in two vertebrae and 27 in three vertebrae (Table 3). All vertebrae with fixation equaled to 151 , the most frequency belonged to L5 (35.8\%) and L4 (31.1\%), respectively. In 62 patients, fixation was performed with screw. Disc evacuation was performed in 55 patients, 8 of which were not necessary. In 47 patients, only one and in 8 patients two discs were evacuated. The sum of evacuated discs was 63. L4-L5 had the highest frequency (Figure 2).

\section{Discussion}

Based on findings of present study, 52 of 63 patients were women $(82.5 \%)$ and 11 men $(17.5 \%)$, which is consistent with the overall prevalence rate that is approximately five times higher in women compared to men. In a study by Ebrahimzadeh et al. (1996) in Iran, 13 patients were women and 7 men (12). Kalpakcioglu et al. (2009) in one study and Ahnand Jhun (2015) in another one reported that the number of women was higher than men $(13,14)$. The most common type of spondylolisthesis was degenerative (95.2\%). Except 3 cases, all others were degenerative. The study by Farzanegan (2006) also showed that the most common type of listhesis was degenerative (15) which might be related to patients' social and cultural conditions, job and residence place. Yet, more comprehensive studies are required to prove this claim. $92.06 \%$ of patients had slip of one vertebra and $7.94 \%$ of them more than one vertebra. L4 and L5 were the most involved vertebrae in women and men, respectively. Denard et al. (2010) found that mean age of men was $74( \pm 6)$. The prevalence of spondylolisthesis was $31 \%$ $(n=92)$ and was observed at L5/S1, L4/L5, L3/L4. In 96\% of cases, only one vertebra had spondylolisthesis (16). Since the main goal of spondylolisthesis surgery is spine decompression, all patients underwent laminectomy $(66.6 \%$ in one vertebra and $33.4 \%$ in more than one) and foraminotomy (36.1\% in one vertebra and $63.9 \%$ in more than one). The most common site of laminectomy was L4 (52.3\%) and the most common site of foraminotomy was L5 (50.5\%). In a study conducted in Canada and Germany, Martin et al. (2007) considered 13 performed studies from 1996 to 2005 on degenerative spondylolisthesis, fusion with decompression of nerve roots that led to better clinical results compared to fusion without it (17). This study was only a general review on spondylolisthesis. Since the articles published in this field in Iran are inadequate, further research with wider scopes seems necessary. Novel studies based on clinical data and therapeutic results, especially developing more intricate research projects based on radiologic findings are suggested.

\section{Conclusion}

In this study, the prevalence of spondylolisthesiswas approximately five times higher in women than in men. The most common type ofspondylolisthesis was degenerative. Most spondylolisthesis slip was observed in one vertebra. Type of surgery was fixation with fusion. The prevalence of spondylolisthesis in operated patients in Poursina Hospital, the surgery method and the common involved sites were consistent with literature.

\section{Acknowledgment}

This article is extracted from MSc thesis (Code, 1516) in Guilan University of Medical Sciences, Iran. We offer our greatest thanks to Clinical Research Development Unit of Poursina Hospital, Guilan University of Medical Sciences, Rasht, Guilan, Iran.

\section{Funding \\ None.}

\section{Conflicts of Interest}

The authors affirm that they have no conflicts of interest.

\section{References}

1.Borenstein David, Wiesel Sam, low back and neck pain:comprehensive diagnosis and management. 3rd ed. Saunders. Usa. 2004

2.Editorial. High-grade lumbosacral spondylolisthesis in children and adolescents: Pathogenesis, morphological analysis, and therapeutic strategy. Joint Bone Spine.2007;74:414-417.

3. Crockard Alan, Hayward Richard, Hoff Julian. Neurosurgery: The Scientific Basis of Clinical Practice, Vol 2. 3rd Ed. Wiley-Blackwell, usa, 1999.

4.M. Kliot, H. Brem, R. Saway. H. Richard Winn. Youmans Neurological Surgery. Vol. 5. 5th Edition, Saunders. USA .2004.

5.Dantas FL, Prandini MN, Ferreira MA. Comparison between posterior lumbar fusion with pedicle screws and posterior lumbar interbody fusion with pedicle screws in adult spondylolisthesis. ArqNeuropsiquiatr. 2007;65:764770 .

6.McTimoney CA, Micheli LJ. Current evaluation and management of spondylolysis and spondylolisthesis. Curr Sports Med Rep. 2003 Feb;2(1):41-6. 7. Watters W, Bono C, Gilbert T et al. An evidence-based clinical guideline for the diagnosis and treatment of degenerative lumbar spondylolisthesis. The Spine Journal.2009;9:609-614.

8. Denard P, Holton K, Miller J et al. Back pain, neurogenic symptoms, and physical function in relation to spondylolisthesis among elderly men. The Spine Journal. 2010;10:865-873.

9. Kent T. Yamaguchi Jr. David L. Skaggs •Daniel C. Acevedo Karen S. Myung Paul Choi Lindsay Andras.Spondylolysis is frequently missed by MRI in adolescents with back pain. J Child Orthop (2012) 6:237-240.

10. Hansdorfer M A, Mardjetko S M, Knott P T, Thompson S E. Lumbar Spondylolysis and Spondylolisthesis in Down Syndrome:A Cross-sectional Study at One Institution. Spine Deformity 1 (2013) 382-388.

11. Ponsa M, Schlenzha D, Seitsalo S et al. Surgical treatment of severe isthmic spondylolisthesis in adolescents:reduction or fusion in situ. Spine. 
1993;18:894-901.

12.Ebrahimzadeh S.A, Etemadifar M. Comparing the results of spondylolisthesis with and without instrument.Journal of Isfahan Medical School. 2002;20(65):51-52.

13. Kalpakcioglu B, Altinbilek T, SenelK.Determination of spondylolisthesis in low back pain by clinical evaluation. J Back MusculoskeletRehabil. 2009;22(1):27-32. doi: 10.3233/BMR-2009-0212.

14. Kang Ahn and Hyung-JoonJhun ,New physical examination tests for lumbarspondylolisthesis and instability: low midline sillsign and interspinous gap change during lumbarflexion-extension motion, BMC Musculoskeletal Disorders (2015) 16:97. DOI 10.1186/s12891-015-0551-0.

15. FarzaneganGh. Lumbar fusion results using pedicle screws in patients with spondylolisthesis. Kowsar Medical Journal. 2006; 11(1):75-80.

16. Denard PJ, Holton Kathleen F, Miller Jessica, et al. Lumbar spondylolisthesis among elderly men: prevalence, correlates, and progression. Spine (Phila $\mathrm{Pa}$ 1976). 2010May 1; 35(10): 1072-1078.

17. Martin CR, Gruszczynski AT, Braunsfurth HA, Fallatah SM, O’Neil J,

Wai EK. The surgical management of degenerative lumbar spondylolisthesis.

A systematic review. Spine. 2007;32:1791-1798. doi: 10.1097/ BRS.0b013e3180bc219e. 